\section{Pause on avian flu transmission studies}

The continuous threat of an influenza pandemic represents one of the biggest challenges in public health. Influenza pandemics are known to be caused by viruses that evolve from animal reservoirs, such as birds and pigs, and can acquire genetic changes that increase their ability to transmit in humans. Pandemic preparedness plans have been implemented worldwide to mitigate the impact of influenza pandemics. A major obstacle in preventing influenza pandemics is that little is known regarding what makes an influenza virus transmissible in humans. As a consequence, the potential pandemic risk associated with the many different influenza viruses of animals cannot be assessed with any certainty.

Recent research breakthroughs identified specific determinants of transmission of $\mathrm{H} 5 \mathrm{~N} 1$ influenza viruses in ferrets. Responsible research on influenza virus transmission using different animal models is conducted by multiple laboratories in the world using the highest international standards of biosafety and biosecurity practices that effectively prevent the release of transmissible viruses from the laboratory. These standards are regulated and monitored closely by the relevant authorities. This statement is being made by the principal investigators of these laboratories.

In two independent studies conducted in two leading influenza laboratories at the University of WisconsinMadison and Erasmus MC in Rotterdam, the Netherlands, investigators have proved that viruses possessing a haemagglutinin (HA) protein from highly pathogenic avian $\mathrm{H} 5 \mathrm{~N} 1$ influenza viruses can become transmissible in ferrets. This is critical information that advances our understanding of influenza transmission. However, more research is needed to determine how influenza viruses in nature become human pandemic threats, so that they can be contained before they acquire the ability to transmit from human to human, or so that appropriate countermeasures can be deployed if adaptation to humans occurs.

Despite the positive publichealth benefits these studies sought to provide, a perceived fear that the ferret-transmissible H5 HA viruses may escape from the laboratories has generated intense public debate in the media on the benefits and potential harm of this type of research. We would like to assure the public that these experiments have been conducted with appropriate regulatory oversight in secure containment facilities by highly trained and responsible personnel to minimize any risk of accidental release. Whether the ferret-adapted influenza viruses have the ability to transmit from human to human cannot be tested.

We recognize that we and the rest of the scientific community need to clearly explain the benefits of this important research and the measures taken to minimize its possible risks. We propose to do so in an international forum in which the scientific community comes together to discuss and debate these issues. We realize that organizations and governments around the world need time to find the best solutions for opportunities and challenges that stem from the work. To provide time for these discussions, we have agreed on a voluntary pause of 60 days on any research involving highly pathogenic avian influenza H5N1 viruses leading to the generation of viruses that are more transmissible in mammals. In addition, no experiments with live H5N1 or H5 HA reassortant viruses already shown to be transmissible in ferrets will be conducted during this time.

We will continue to assess

the transmissibility of H5N1

influenza viruses that emerge

in nature and pose a continuing

threat to human health.

Ron A. M. Fouchier Erasmus

$M C$, Rotterdam, the Netherlands.

Adolfo García-Sastre Mount

Sinai School of Medicine,

New York, USA.

Yoshihiro Kawaoka University

of Tokyo, Japan; and University of

Wisconsin-Madison, Wisconsin,

USA.

kawaokay@svm.vetmed.wisc.edu For a full list of co-authors, see go.nature.com/mwqvoo

\section{Train local experts to help conserve forests}

The management of tropical

forests by indigenous

communities requires a formal

intervention plan based on

quantitative inventories,

growth studies and permanent

monitoring systems. But in our

30 years of experience, few such

communities, if any, would be

able to produce a plan on their

own. This shortcoming needs to

be rectified.

One answer would be to train village-based resource managers, who could use their knowledge and skills in forestry, ecology, ethnobotany, economics and anthropology to help local communities manage forests and interact with the state, commercial markets and the global conservation community. This would reduce dependency on external inputs, draw on local skills, promote community organization and self-governance, and help to implement ways of reducing emissions from deforestation and degradation.

Crucially, community-owned forests would then stay in the hands of those who have lived in, used and looked after them for so long.

Charles M. Peters The New York Botanical Garden, New York, USA. cpeters@nybg.org

Miguel Alexiades University of
Kent, Canterbury, UK. Sarah A. Laird People and Plants International, Bristol, Vermont, USA.

\section{Biobank donors should have a say}

Our findings from a qualitative survey of patients, health-care providers, researchers and other members of the public in Switzerland support suggestions that the views of prospective tissue donors should be taken into account in securing biobank samples (Nature 478, 312-313; 2011).

In 2009-10, we interviewed 90 people in focus groups, using fictitious case studies to explore perceptions about the use of human tissue.

Those professionals who preferred blanket consent from donors commonly expressed fears that providing information on tissue use to patients could make them reluctant to donate samples. The general population and patient groups, by contrast, usually wanted to be informed and have the opportunity to give consent.

Among patients, the need to be informed was linked to a wish to be appraised of their medical condition and to be a partner in the therapeutic process. Cancer patients were particularly enthusiastic about the use of their tissues because it helped them to make sense of their illness and could potentially benefit others. Brenda Spencer, Daria Koutaissoff, Hans-Anton Lehr Lausanne University Hospital, Switzerland. brenda.spencer@chuv.ch

\section{CONTRIBUTIONS}

Correspondence may be sent to correspondence@ nature.com after consulting the guidelines at go.nature. com/cmchno. Readers can also comment online: www.nature.com/nature. 